\title{
In silico molecular GRIP docking of some secondary metabolites combating diabesity
}

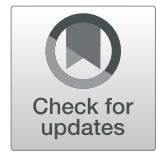

Baishakhi De ${ }^{1 \dagger}$, Koushik Bhandari $^{1 \dagger}$, Rajeev K. Singla ${ }^{2}$, Gargi Saha ${ }^{3}$ and Tridib Kumar Goswami ${ }^{*^{*}}$ (D)

\begin{abstract}
Background: This research article aims to study at molecular level by in silico GRIP docking the effectivity of the detected compounds in value added black tea or VABT, prepared in a chemometrically optimized ratio of Black tea: Cinnamon (Cinnamomum zeylanicum):Ginger (Zingiber officinale):Cardamom (Elettaria cardamomum) in combating diabesity.

Results: The compounds in VABT were identified by liquid chromatography-mass chromatography. Basing on the desirability function, the chemometrically optimized weight ratio of VABT was determined to be 420:50:54:26 (Black tea:cinnamon:ginger:cardamom). Among the identified molecules, phlorizin, 6-gingerol, procyanidin, and epicatechin were docked with insulin receptor kinase, pancreatic lipase-colipase complex, and human FTO. The high negative dock scores of the test molecules in inhibiting the enzymes suggest their potency.

Conclusions: Thus, the results of in silico studies predicted the potency of VABT in combating diabesity. VABT thus has potency and can serve as a nutrotherapeutic entity against diabesity.
\end{abstract}

Keywords: Value added black tea, Phlorizin, 6-Gingerol, Procyanidin, Epicatechin, GRIP docking, Diabesity

\section{Background}

The incidence of "nutrition transition" increase work place stress; involvement in sedentary leisure time has played a great role in increased incidence of chronic diseases. Diabesity is the combined epidemic of type 2 diabetes and obesity. This lifestyle disease is affected by a multitude of pathogenic factors viz. lipotoxicity, oxidative stress, and disturbances in leptin levels. Almost all available treatment options with thiazolidinediones, sulphonylureas, meglitinides, and insulin are associated with weight gain as one of the side effects. Approved anti-obesity drugs are also not free of side effects. Liposuction by surgical procedure or bariatric surgery is not a viable option in the Indian context (McNaughton 2013; Belavic 2015). The changing landscape of treatment strategy has focused considerably on therapeutic

\footnotetext{
* Correspondence: tkg@agfe.iitkgp.ernet.in

Baishakhi De and Koushik Bhandari equally contributed and are jointly regarded as first authors.

${ }^{1}$ Department of Agriculture and Food Engineering, Indian Institute of Technology, Kharagpur 721302, India

Full list of author information is available at the end of the article
}

lifestyle changes that refer mostly to dietary interventions, increase in physical activities, avoidance of sedentary lifestyle, etc. along with pharmacotherapy (Mitra et al. 2012). The incorporation of integrative medicine that hyphenates the evidence-based complementary therapy with the conventional approaches is now being widely implemented in treating several chronic ailments (De et al., 2019a, b; Medagama 2015). Research evidences have shown the great adjunct potentialities of plant bioactives as multi target entities in treating several multifactorial ailments. However, their nutro-health effects can be maximally exploited on identification of potential molecular targets. Plant secondary metabolites (PSMs) either as nutraceuticals or dietary supplements mostly in combinations or combinatorics are found to show synergistic nutrotherapeutic effects (Mohamed et al. 2014; De et al., 2019a, b; De et al., 2015a, b). Value addition in nutraceuticals, food combinatorics, and multi-component concentrates are already in vogue. Diversification products of the popular beverage tea 
(Camellia sinensis) have already attained the global attention (Ghosh and Yang 2015).

Successful implementation of bioinformatics tools for the fast and frugal screening of pharmacologically active compounds from the complex matrix of medicinal plants has reduced labor, cost, and time. GRIP ${ }^{\mathrm{Tw}}$ is a novel methodology available as rigid as well as flexible docking for rapid, accurate protein-peptide and proteinligand docking as studied by the $\mathrm{V}$ Life software. The aim of protein-ligand docking is to calculate the binding energy of the protein-ligand reaction complex at given atomic coordinates. The key parameters for flexible docking include energy functions, protein catalytic sites, and active residues. It makes use of a set of ligands with its conformers to be docked into the receptor cavity. This software helps to search for the active sites, consists of pre-computation of grids, and tries to maximize favorable interaction and minimize unfavorable and repulsive interaction by proving the best possible orientation. GRIP-scoring function helps in quick and precise determination of ligand-receptor interactions in the active sites of proteins. In GRIP docking, unique conformers of a set of ligands are considered as input and offer the advantages of wide range of parameterizations, both ligand-guided as well as cavity-guided docking options, consider hydrogen bonding, repulsions, and dispersion interactions in different modes of operations, e.g., manual, automated, and batch mode (De et al., 2017a, b; Bhandari et al. 2015). Literature mining reported the role of docking studies in screening of phytomolecules exhibiting different pharmacological actions (Meruva et al. 2014; Jadhav et al. Singla and Bhat, 2010a, b; Singla et al. 2013; Jawla and Kumar 2013; Malleshappa and Patel 2013; Kaushik et al. 2014; Mahendran et al. 2014; Igoli et al., 2014a, b, c, d).

This research article aims to study at molecular level by in silico GRIP docking the effectivity of the detected compounds in value added black tea or VABT, prepared in a chemometrically optimized ratio of Black tea:Cinnamon (Cinnamomum zeylanicum):Ginger (Zingiber officinale):Cardamom (Elettaria cardamomum) in combating diabesity.

\section{Materials}

\section{Chemicals and reagents}

All reagents used in the research were of analytical and HPLC grade and were purchased from Merck (India) and Sigma Aldrich (India).

\section{Instruments}

Instruments used were HPLC (Model Waters 2998); FTIR (Nicolet 6700, Thermo Fischer Scientific); and ${ }^{1} \mathrm{H}$ and ${ }^{13} \mathrm{C}$ NMR (Bruker NMR Avance).

\section{Software}

Chemometrics was done by Design Expert Trial version 7.0; GRIP docking studies was done using the MDS V Life software.

\section{Preparation of value added black tea (VABT)}

The mixture design approach of response surface methodology (RSM) was applied to optimize the ratios of the value added adjuvants with black tea for the synergistic attainment of anti-diabesity effect. The ratios of the adjuvants with black tea have been optimized by mixture design of RSM. Actually, the mixture design of RSM holds good while working for optimizations with a mixture or food components, chemical compositions or drug formulations (Simon et al. 1999). VABT can be considered as an edible food mixture. In any experiment, mixture design is chosen when the components are added to a fixed total. If the percentage of one component is increased, then the percentage of other components is to be decreased, and the response must be the function of the proportion of the components. Mixture approach offers the advantage of defining the experimental region of interest more naturally (Simon et al. 1999).

With the help of Design Expert Trial version 7.0, the entire RSM for optimizing the ratios of VABT was carried out. Software-based suggested and statistically significant models have been chosen. Oxygen radical absorbance capacity (ORAC) and organoleptic scores are the independent variables so as to optimize the ratio of VABT with components Black tea, cinnamon, ginger, and cardamom.

Preparation of VABT Basing on the chemometrically optimized weight ratio of 420:50:54:26 (Black tea:cinnamon:ginger:cardamom), the total mixture was subjected to hot water maceration for $3 \mathrm{~h}$ and then subjected to filtration. The filtrate was centrifuged at $4000 \mathrm{rpm}$ for 10 min, and the supernatant was collected and concentrated under rota evaporation. The concentrated mass was dried in hot air oven at $50{ }^{\circ} \mathrm{C}$ in sterile petri dishes, and the dried mass scraped out in powdered form and stored in air tight containers.

\section{Chemo profiling of VABT by liquid chromatography-mass spectroscopy}

Chemo profiling of VABT was done by LC-MS using $0.2 \%$ acetic acid and acetonitrile at a flow rate of $1 \mathrm{ml} /$ min. Mass spectrometer with the capability of electrospray ionization (ESI) switching between the positive and negative polarity conditions were used. ESI negative modes were used for detecting the catechins and ESI positive mode for detecting the theaflavins. The capillary voltage was maintained at $3 \mathrm{kV}$, cone voltage at $30 \mathrm{~V}$, 
and extractor voltage at $4 \mathrm{~V}$ for both positive and negative mode to achieve the best sensitivity for the analytes. The source temperature and desolvation temperature were maintained at $150{ }^{\circ} \mathrm{C}$ and $625^{\circ} \mathrm{C}$ respectively. The cone gas flow rate was kept at $50 \mathrm{~L} / \mathrm{h}$ and that of salvation gas flow rate at $1100 \mathrm{~L} / \mathrm{h}$ (Ganguly et al. 2016).

\section{Spectral identification by Fourier transform infrared spectroscopy}

For FTIR studies, VABT was properly blended with $\mathrm{KBr}$. Then $\mathrm{KBr}$ pellets were prepared and scanned in the range of $4000-400 \mathrm{~cm}^{-1}$. A resolution of $4 \mathrm{~cm}^{-1}$ was used.

\section{In silico GRIP docking studies}

In order to understand at molecular level how the nutrotherapeutic product VABT is exerting its antidiabesity effect, a very robust software V Life MDS version 4.6 with inclusion of all the necessary simulation modules were applied for the same (V Life MDS 2013).

\section{Target proteins used}

3EKK is an insulin receptor kinase complexed with an inhibitor. Inhibitor is $2-[(2-\{[1-(\mathrm{N}, \mathrm{N}-$ dimethylglycyl $)-5-$ methoxy- $1 \mathrm{H}$-indol-6-yl] amino\}-7H-pyrrolo [2,3-d] pyrimidin- 4-yl) amino]-6-fluoro-N-methylbenzamide. Source for the protein is human [https://www.rcsb.org/ $\mathrm{pdb} /$ explore/explore.do?structureId=3EKK]. $\mathbf{1 N 8 S}$ is a pancreatic lipase-colipase complex. Co-enzyme is Colipase II. Source for the protein is human [http://www. rcsb.org/structure/1N8S]. $4 \mathrm{CXW}$ is a human FTO in complex with subfamily-selective inhibitor 12 . Inhibitor is (2E)-4-[N'-(4-benzyl-pyridine-3-carbonyl)-hydrazino]4-oxo-but-2-enoic acid. Source for the protein is human [http://www.rcsb.org/structure/4CXW].

\section{Docking studies}

The necessary data and information about the structure of these proteins was obtained from RCSB Protein Data Bank (PDB). VLife MDS version 4.6 was used for current docking study (Pokuri et al. 2014; www.vlifesciences. com). The 2D structures of the active compounds in VABT (e.g., phlorizin, epicatechin, 6-gingerol, procyanidin) as detected and confirmed in LCMS studies were drawn using ChemDraw Ultra 8.0 and further exported it to the VLife MDS 4.6. Energy minimization was done using Merck Molecular Force Field (MMFF) and MMFF atomic charge with 1.0 constant as distance-dependent dielectric function, 10,000 maximum number of cycles, 0.01 as root mean square (rms) gradient, and gradient numerical followed by analytical. For docking validation, the reference ligand was re-docked just like other test molecules. The parameters fixed for docking simulation was like this: number of placements, 100; rotation angle, $10^{\circ}$; exhaustive method; ligand-wise results, 10; scoring function: dock score. By rotation angle, ligands would be rotated inside the receptor cavity to generate different ligand poses inside the receptor cavity. By placements, the method will check all the 100 possible placements into the active site pocket and will result out best placements out of 100. After docking simulation, the best docked conformer of test ligands and reference ligand were then checked for their interactions with targeted proteins like hydrogen bonding, hydrophobic, pi-staking/ aromatic, charge and van der Waal's interactions (De et al., 2015a, b; Ghosh and Yang 2015; De et al., 2017a, b; Bhandari et al. 2015; Meruva et al. 2014; Jadhav et al.; Singla and Bhat, 2010a, b; Singla et al. 2013; Jawala and kumar 2013; Malleshappa and Patel 2013; Koushik et al. 2014; Mahendran et al. 2014; Igoli et al., 2014a, b, c, d).

\section{Results}

Considering the results of RSM for optimizing the ratios of additives with black tea, the process order fits to quadratic design model with ORAC values as response 1 and organoleptic scores as response 2 . With both responses (1 and 2), the normal plot of residuals helps to measure the number of standard deviations separating the actual and predicted values, and the normality assumption was satisfied with very less scattering of points and fitted almost to a straight line (Fig. 1). The model summary statistics focus on the model maximizing the "adjusted R-squared" and "predicted R-squared" values. The "R-squared" values of 0.9936 (response 1) and 0.9870 (response 2) having closeness to unity showed a good fitness between the actual and that obtained from the response model. The desirability values for the responses are shown in (Fig. 2). The dot on each ramp function graph indicates the optimal level of the parameter. The desirability function value ranges from zero, outside of the limits, to one at the goal; the purpose of the program is to maximize the function; seeking of goal begins at a random starting point and proceeds up the steepest slope to a maximum.

The LC-MS chromatogram of VABT (Fig. 3) showed the presence of several compounds; however, those with good abundances were detected within the retention time range of $1.2-31.68 \mathrm{~min}$.

The presence of compounds were confirmed both by chromatographic and MS data and also comparison with retention times as well as UV-vis spectra and maximum absorption wavelength (Fig. 4). Tea catechins were detected in the ESI negative mode, and the powerful black tea antioxidants, the theaflavins, were detected in the ESI positive mode.

Tea catechins were detected in the ESI negative mode, and the powerful black tea antioxidants the theaflavins were detected in the ESI positive mode, within the mass 


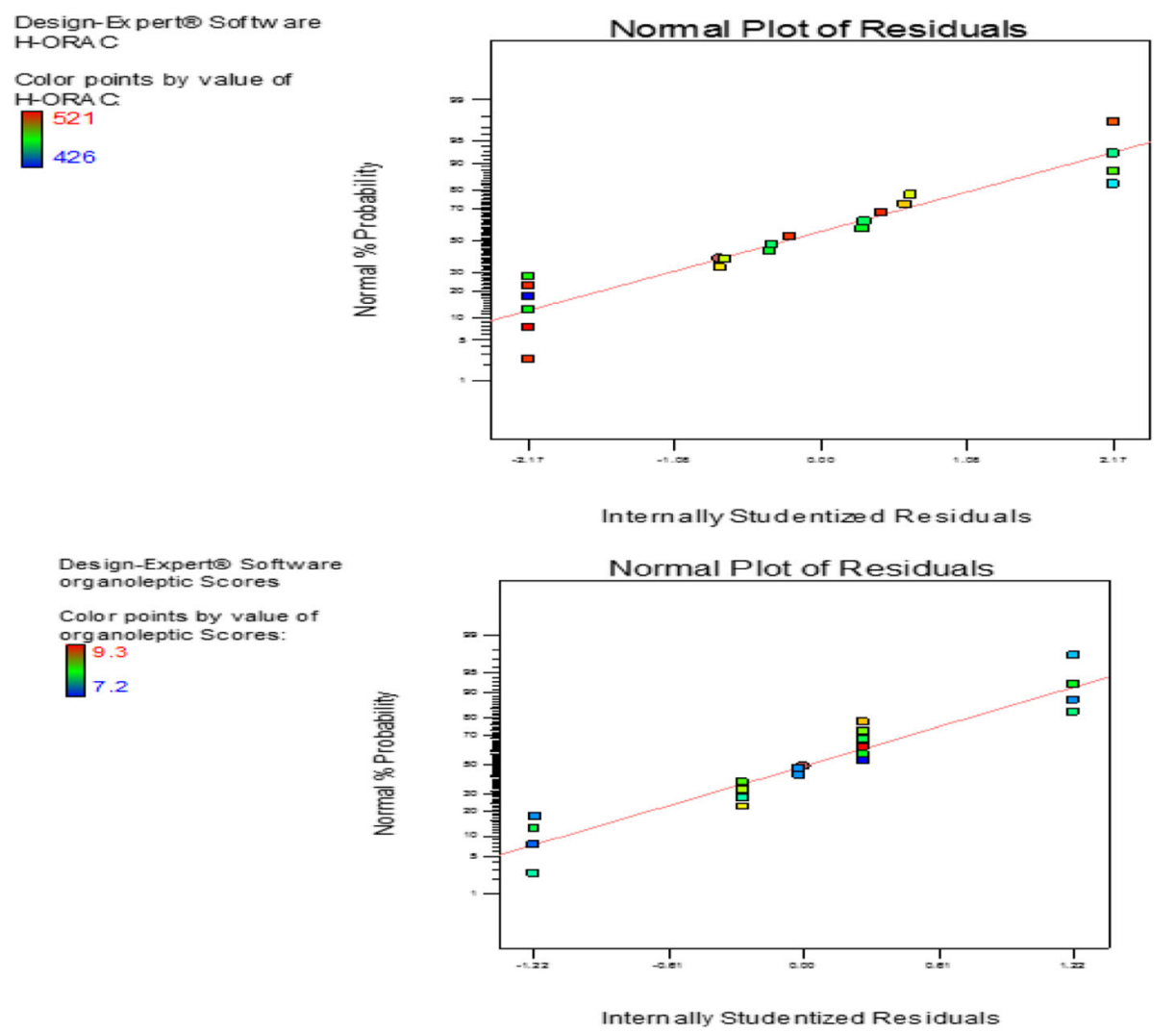

Fig. 1 Normal plot of residuals with ORAC value (above) as response 1 and organoleptic score (below) as response 2

range of $100-1000 \mathrm{~m} / \mathrm{z}$ quercetin derivatives, tea catechins, theaflavins, kaempferol -3-O- glucoside (Fig. 5). The detected compounds include gallic acid $(\mathrm{m} / z$ 169), caffeine $(\mathrm{m} / z 195)$, catechin and epicatechin $(\mathrm{m} / z 289)$, gallocatechins and epigallocatechins $(\mathrm{m} / z \mathrm{305})$, gallocatechin gallate and epigallocatechin gallate $(m / z 457)$, and catechin gallate and epicatechin gallate $(\mathrm{m} / \mathrm{z} 441)$. In addition, VABT showed the presence of cinnamaldehyde $(\mathrm{m} / \mathrm{z} 133)$, coumarins $(\mathrm{m} / \mathrm{z} 147)$, and the polymerized polyphenols, the procyanidins. Proanthocyanidins are various length polymers of flavanols (catechins and their ent-isomers) linked through a single $\mathrm{C} 4 \rightarrow \mathrm{C} 8$ or $\mathrm{C} 4 \rightarrow$ $\mathrm{C} 6$ bond (B-type) or with an additional $\mathrm{C} 2 \rightarrow \mathrm{O} \rightarrow \mathrm{C} 7$ or $\mathrm{C} 2 \rightarrow \mathrm{O} \rightarrow \mathrm{C} 5$ bond (A-type). There are two main varieties of procyanidin oligomers that occur in plants. These can be distinguished from each other by mass spectrometric analysis. For example, the A-type procyanidin trimers and tetramers have molecular masses of 864 and $1152 \mathrm{Da}$, whereas the comparable B-type procyanidin trimers and tetramers have molecular masses of 866 and $1154 \mathrm{Da}$, respectively. Type A trimer proanthocyanin $(\mathrm{m} / z \mathrm{865})$, tetramers $(\mathrm{m} / \mathrm{z} 1153.2629)$, pentamers $(\mathrm{m} / z$ 1441.3259), type B trimers $(\mathrm{m} / \mathrm{z}$ 867.2134), and tetramers $(m / z 1155.2782)$ were detected in VABT.
In addition, the MS spectra of several compounds eluted at different $t_{R}$ showed the presence of several gingerol related compounds. 4, 6, and 8-gingerols were identified within the retention times of 25.6, 32.6, and $38.7 \mathrm{~min}$, detected both in negative and positive ESI mode with the corresponding $\mathrm{m} / z$ values of $\mathrm{m} / z 265$ and 249 for 4-gingerol, $m / z 293$ and 277 for 6-gingerol, $m / z$ 321 and 305 for 8-gingerol; 6-shogaol was eluted at 35.8 min with $m / z 275$ and 277 in both negative and positive ESI mode. 6-Paradol eluted at $38.2 \mathrm{~min}$ was detected in positive ESI mode $(m / z 279)$.

\section{Spectral interpretation}

As shown above in LCMS studies, VABT is a matrix of several active compounds, and thus, the resultant spectra is a finger print of the presence of different molecules. The spectrum indicated the presence of aliphatic $\mathrm{O}-\mathrm{H}$ groups in the range of 3000 to $3600 \mathrm{~cm}^{-1}$. The bands at $2800-2950 \mathrm{~cm}^{-1}$ displayed two stretching peaks; $\mathrm{CH}_{2}$ and $\mathrm{CH}_{3}$ are well separated (2944 and $2832 \mathrm{~cm}^{-1}$ ); band at $1030 \mathrm{~cm}^{-1}$ indicated the presence of $\mathrm{C}-\mathrm{O}$ group. $\mathrm{N}-$ $\mathrm{H}, \mathrm{O}-\mathrm{H}, \mathrm{C}=\mathrm{C}$, and $\mathrm{C}=\mathrm{O}$ stretching peaks were observed at $3387.5 \mathrm{~cm}^{-1}, 3677.5 \mathrm{~cm}^{-1}, 1652.5 \mathrm{~cm}^{-1}$, and 1517.5 $\mathrm{cm}^{-1}$, respectively. FTIR bands $\left(\mathrm{cm}^{-1}\right)$ were observed at 


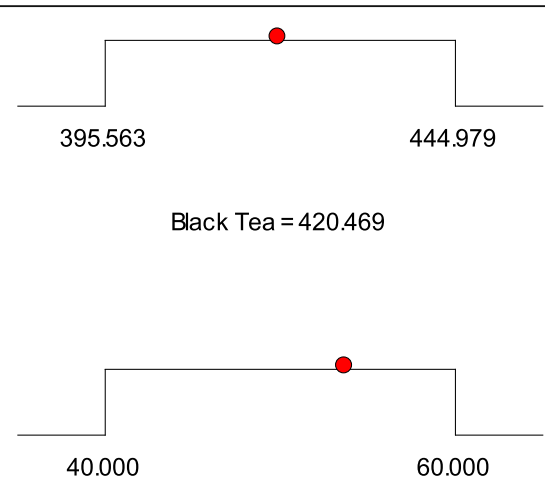

Ginger $=53.873$

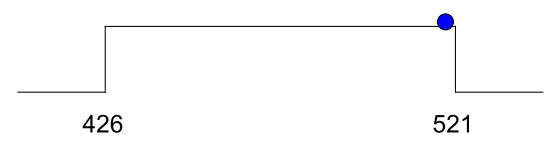

$\mathrm{H}-\mathrm{ORAC}=519.575$

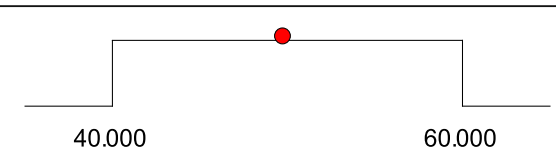

Cinnamon $=49.978$

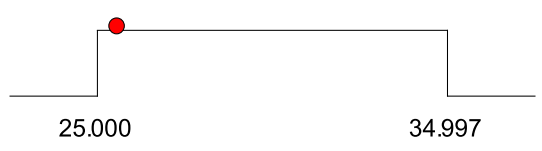

Cardamom $=25.680$

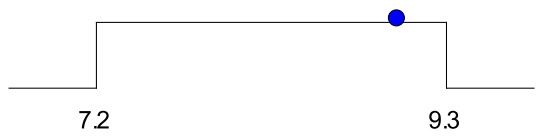

organoleptic Scores $=9.02677$

Desirability $=1.000$

Fig. 2 Desirability ramp function graph

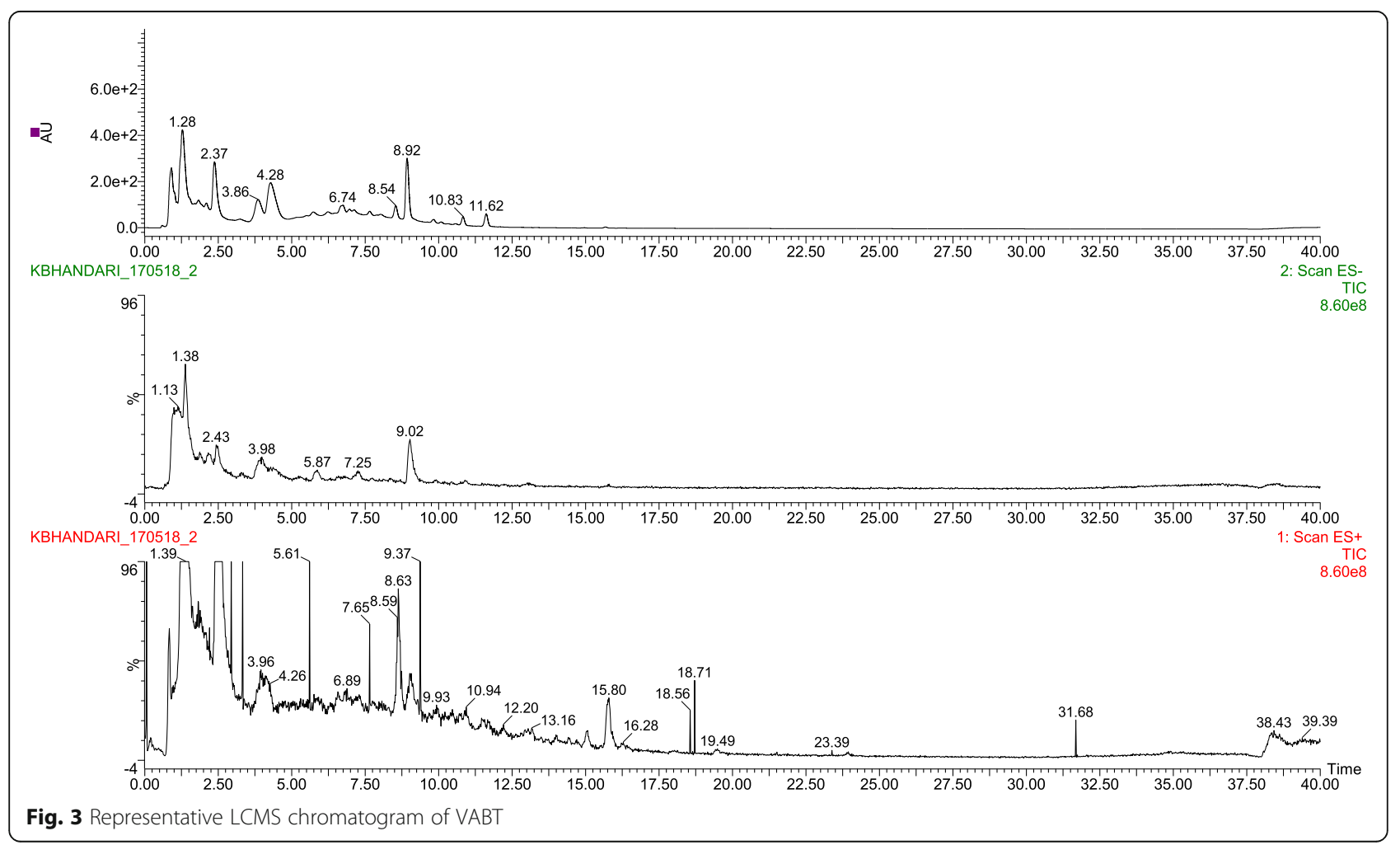




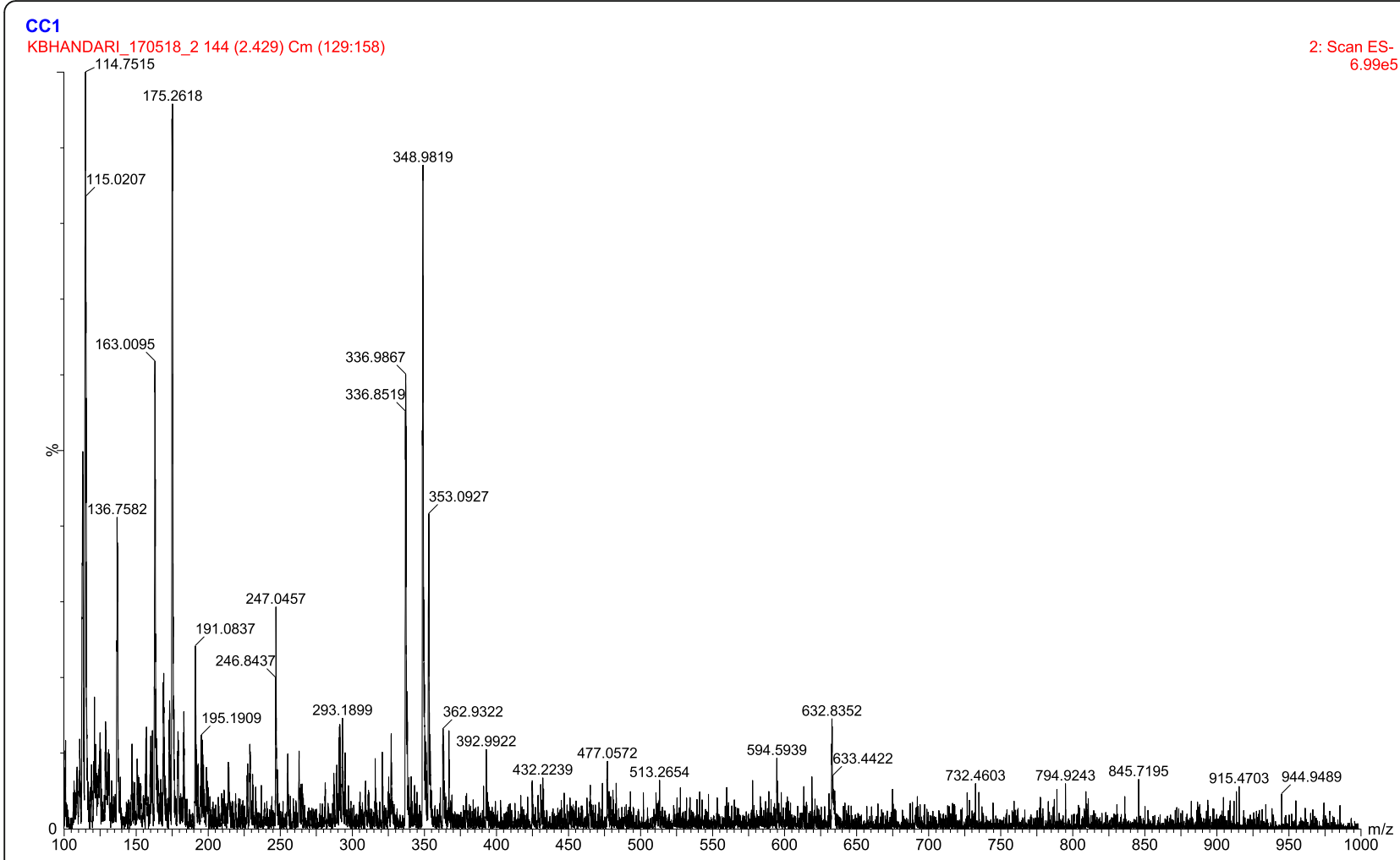

Fig. 4 Representative MS spectra in negative mode of compounds in VABT eluted at 2.4 min retention time

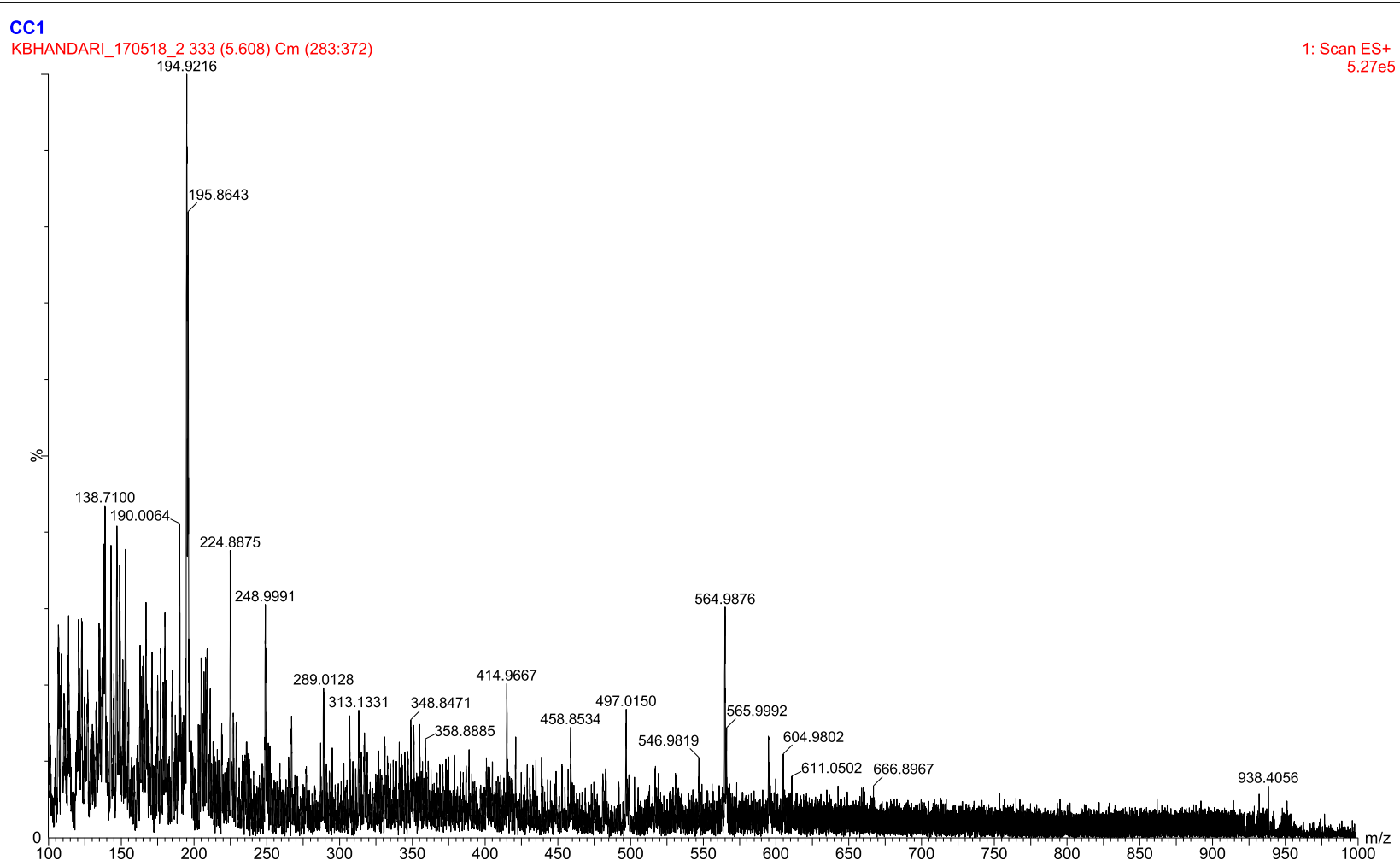

Fig. 5 Representive MS spectra in positive mode of compounds in VABT eluted at 5.6 min retention time 
$3383(\mathrm{OH}), 1640(\mathrm{C}=\mathrm{O}), 1580,1450(\mathrm{Ar}), 1200$ (Ar-O$\mathrm{C})$, and 1087 (C-OC) that matched with the standard FTIR spectra of phlorizin. The polymers of procyanidin type mostly show single peaks at $1520-1540 \mathrm{~cm}^{-1}$ and absorption peak at $770-780 \mathrm{~cm}^{-1}$. VABT showed the presence of strong absorption peaks at $2920 \mathrm{~cm}^{-1}$ and $1150 \mathrm{~cm}^{-1}$ showing the presence of procyanidins. Some catechins gave bands at $1519 \mathrm{~cm}^{-1}$; the methyl xanthenes also showed their characteristic IR bands; the CO stretch due to imide functionality was observed at $1690 \mathrm{~cm}^{-1}$ showing the presence of theobromine; the two bands in the carbonyl region, the one at $1700 \mathrm{~cm}^{-1}$ due to the $\mathrm{CO}$ stretch of the disubstituted amide and the other band at $1656 \mathrm{~cm}^{-1}$ due to CO stretch of urea indicated the presence of caffeine.

\section{Docking studies}

Comparison of docking affinity of the pharmacologically active molecules with the ligand based cocrystallized site of the targeted proteins (Table 1) have shown that the highest negative dock score was shown by the reference ligand. However, the high negative dock scores of the identified molecules in VABT, e.g., phlorizin (-86.04), 6gingerol (-79.33), and epicatechin (-82.53), in inhibiting the target protein $4 \mathrm{CXW}$ (human alpha-ketoglutaratedependent dioxygenase FTO) also showed their effectivity in comparison to the reference ligand. Procyanidin was also found to be a potent molecule in inhibiting the target proteins 3EKK and 1N8S (Table 1). Gingerol also showed significant potency in inhibiting 3EKK and $1 \mathrm{~N} 8 \mathrm{~S}$.

Considering the target protein, human insulin receptor kinase (3EKK, PDB) was used to study the interactions between the test molecules and the human insulin receptor kinase, and it was compared with the reference ligand which is $2-[(2-\{[1-(\mathrm{N}, \mathrm{N}-$ dimethylglycyl $)-5$-methoxy-1H-indol-6-yl] amino\}-7H-pyrrolo [2,3-d]

Table 1 Comparison of docking affinity of the test molecules with the ligand based cocrystallized site of the targeted proteins

\begin{tabular}{llll}
\hline Detected compounds & \multicolumn{3}{l}{ Scoring function ${ }^{\mathrm{a}}$} \\
\cline { 2 - 4 } & 3EKK & 1N8S & 4CXW \\
\hline Phlorizin & -77.76 & -62.33 & -86.04 \\
6-Gingerol & -61.79 & -53.47 & -79.33 \\
Procyanidin & -69.76 & -71.20 & -30.36 \\
Epicatechin & -53.04 & -45.58 & -82.53 \\
Corresponding reference ligand $^{\mathrm{b}}$ & -104.08 & -72.27 & -86.16 \\
Corresponding reference ligand $^{\mathrm{C}}$ & -104.08 & $-72.95^{* *}$ & $-86.42^{* * *}$
\end{tabular}

${ }^{*}$ RMSD in this case is 0.5 ; ${ }^{*}$ RMSD in this case is 0.025 ; ${ }^{* *}$ RMSD in this case is 0.004

${ }^{\text {a PLP score }}$

${ }^{\mathrm{b}}$ Dock score obtained when co-crystallized ligand was acting as tracker

${ }^{\mathrm{C}}$ Dock score obtained when co-crystallized ligand was acting as test molecule pyrimidin- 4-yl) amino] -6-fluoro-N-methylbenzamide. Among the test molecules, phlorizin and procyanidin are still the promising one in context to the affinity. Phlorizin has van der Waal's interactions with Leu1002A, Gly1003A, Gln1004A, Gly1005A, Val1010A, Ala1028A, Lys1030A, Val1060A, Leu1078A, Met1079A, Ala1080A, Gly1082A, Asp1083A, and Met1139A while having hydrophobic interactions with Leu1002A, Gly1003A, Gln1004A, Val1010A, Ala1028A, Leu1078A, Met1079A, Gly1082A, Asp1083A, and Met1139A amino acid residues of active site of $3 E K K$ which was tracked by the cocrystallized ligand. 6-Gingerol has van der Waal's interactions with Leu1002A, Gly1003A, Gln1004A, Val1010A, Ala1028A, Lys1030A, Val1060A, Met1076A, Met1139A, Gly1149A, and Asp1150A while having hydrophobic interactions with Gly1003A, Gln1004A, Gly1005A, Val1010A, Ala1028A, Lys1030A, Met1076A, and Met1139A amino acid residues of 3EKK's active site.

Procyanidin has van der Waal's interactions with Leu1002A, Gly1003A, Gln1004A, Gly1005A, Val1010A, Gly1082A, Asp1083A, Arg1136A, Asn1137A, and Met1139A while having hydrophobic interactions with Gly1003A, Gln1004A, Gly1005A, and Asp1083A amino acid residues of active site of the human insulin receptor kinase. Along with that, procyanidin has hydrogen bonding with Gln1004A at a bond distance of $2.030 \AA$. Epicatechin has van der Waal's interactions with Leu1002A, Gly1003A, Gln1004A, Gly1005A, Val1010A, Leu1078A, Ala1080A, Gly1082A, Asp1083A, and Met1139A while having hydrophobic interactions with Gly1082A and Met1139A amino acid residues of active site of 3EKK. Reference ligand, 2-[(2-\{[1-(N,N-dimethylglycyl)-5-methoxy-1H-indol-6-yl]amino\}-7H-pyrrolo[2,3-d]pyrimidin4-yl)amino]-6-fluoro- $\mathrm{N}$-methylbenzamide, has van der Waal's interactions with Leu1002A, Gly1003A, Gln1004A, Gly1005A, Ser1006A, Val1010A, Arg1026A, Ala1028A, Val1060A, Met1076A, Glu1077A, Leu1078A, Met1079A, Ala1080A, Gly1082A, Asp1083A, Ser1086A, Met1139A, and Asp1150A while having hydrophobic interactions with Leu1002A, Gly1003A, Gln1004A, Gly1005A, Val1010A, Glu1012A, Leu1078A, Ala1080A, Gly1082A, Asp1083A, Ser1086A, Met119A, and Asp1150A amino acid residues of active site of 3EKK. Reference ligand also charge interaction with Asp1083A at a bond distance of $2.748 \AA$ while having hydrogen bonding with Glu1077A and Asp1083A at a bond distance of 1.916 and $2.453 \AA$ respectively and with Met1079A at bond distance of 1.984 and $2.071 \AA$ (Fig. 6).

Human pancreatic lipase (1N8S PDB) was used to study the interactions between the test molecules and the human pancreatic lipase, and it was compared with the reference ligand which is colipase II. Colipase II is a small protein co-factor which facilitates pancreatic lipase 


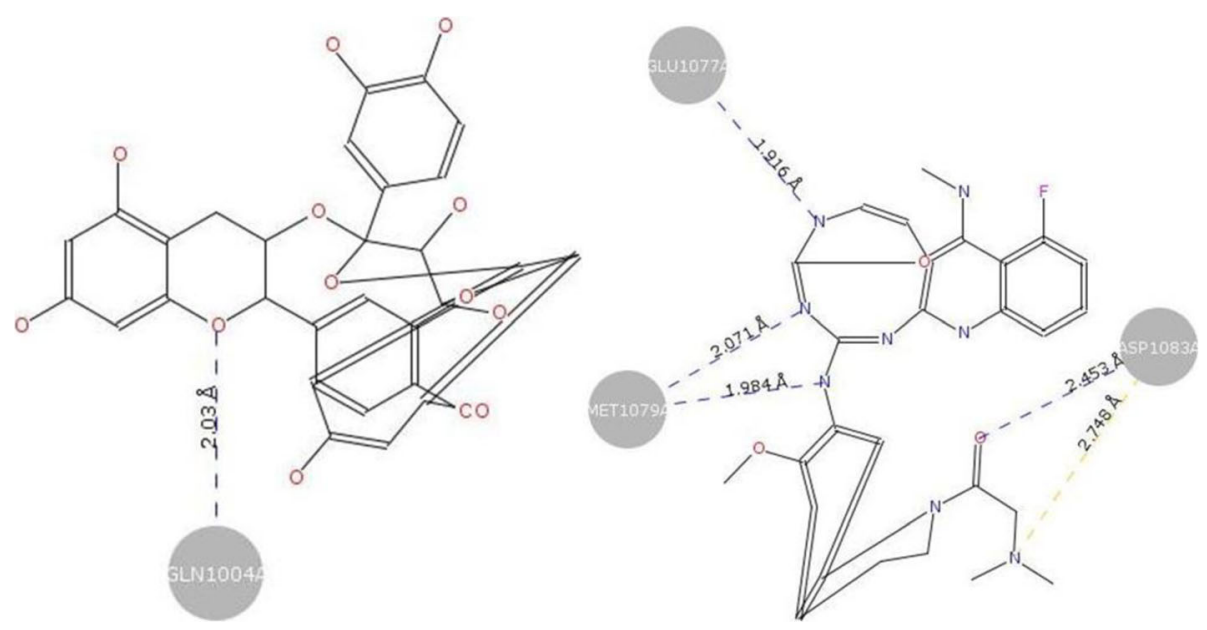

Fig. 6 Hydrogen bonding of procyanidin with cocrystallized ligand-tracked active site in 3EKK (left). Hydrogen bonding and charge interactions of reference ligand with cocrystallized ligand tracked active site in 3EKK (right)

for its optimal activity. With reference to Table 1, it was observed that the procyanidin have comparable affinity for the target protein as compared to the reference ligand. Phlorizin has van der Waal's interactions with Leu356A, Lys367A, Gln368A, Glu370A, Ile401A, Tyr403A, and Glu441A while having hydrophobic interactions with Leu356A, Lys367A, Gln368A, and Tyr369A amino acid residues of active site of human pancreatic lipase, 1N8S, which was tracked by the cocrystallized ligand. Along with that, phlorizin has aromatic interaction with Tyr403A at a bond distance of $4.710 \AA$ while having hydrogen bonding with Lys367A and Glu370A at a bond distance of 2.342 and $1.343 \AA$ respectively. 6-Gingerol has van der Waal's interactions with Leu356A, Phe360A, Asn365A, Ser366A, Lys367A, Gln368A, Tyr369A, Glu370A, and Tyr403A while having hydrophobic interactions with Leu356A, Asn365A, Lys367A, and Gln368A amino acid residues of active site of 1N8S. 6-Gingerol also has hydrogen bonding with Gln368A at a bond distance of $1.855 \AA$ and with Tyr403A at bond distance of 2.464 and 2.599 A. Procyanidin has van der Waal's interactions with Leu356A, Lys367A, Gln368A, Tyr369A, Glu370A, Phe386A, and Tyr403A while having hydrophobic interactions with Leu356A, Lys367A, Gln368A, Tyr369A, and Glu370A amino acid residues of active site of human pancreatic lipase. Procyanidin also has aromatic interaction with Tyr403A at a bond distance of 4.914 $\AA$ while having hydrogen bonding with Lys367A at a bond distance of $2.003 \AA$. Epicatechin has van der Waal's interactions with Lys367A, Gln368A, Tyr369A, Glu370A, Phe386A, and Asp387A while having hydrophobic interactions with Leu356A, Lys367A, Tyr369A, and Glu370A amino acid residues of active site of 1N8S. Apart from that, epicatechin has aromatic interaction with Tyr369A at a bond distance of $4.608 \AA$ while having hydrogen bonding with Lys367A and Glu370A at a bond distance of 1.329 and $2.000 \AA$ respectively. Reference ligand, colipase II, has van der Waal's interactions with Phe360A, Asn365A, Ser366A, Lys367A, Gln368A, Asp389A, Val390A, Lys399A, Ile401A, Tyr403A, Glu441A, and Leu443A while having hydrophobic interactions with Leu356A, Ser366A, Lys367A, Gln368A, Lys399A, Ile401A, Tyr403A, Glu441A, and Leu443A amino acid residues of $1 \mathrm{~N} 8 \mathrm{~S}$. Colipase II also has charge interactions with Lys399A, Asp389A, and Glu441A at a bond distance of $2.945,4.740$, and $3.477 \AA$ respectively while having hydrogen bonding with Asp389A at bond distance of 1.866 and $2.446 \AA$ and with Glu441A at a bond distance of $2.486 \AA$ (Fig. 7).

$4 C X W$ PDB was used to study the interactions between the test molecules and the human alphaketoglutarate-dependent dioxygenase FTO, and it was compared with the reference ligand which is (2E)-4-[N'(4-benzyl-pyridine-3-carbonyl)-hydrazino]-4-oxo-but-2enoic acid. With reference to Table 1 , it was observed that the phlorizin, 6-gingerol, and epicatechin have comparable affinity for the target protein as compared to the reference ligand. Phlorizin has van der Waal's interactions with Thr92A, Pro93A, Val94A, Arg96A, Tyr106A, Tyr108A, Leu109A, Ala227A, Val228A, Ser229A, Trp230A, His231A, His232A, Asp233A, Glu234A, and Arg322A while having hydrophobic interactions with Thr92A, Pro93A, Val94A, Leu109A, Val228A, Ser229A, His231A, and Glu234A amino acid residues of active site of $4 \mathrm{CXW}$ which was tracked by the reference ligand. Along with that, phlorizin has aromatic interactions with Tyr108A and His231A at a bond distance of 5.423 and $4.625 \AA$ respectively while having hydrogen bonding with Arg96A and Tyr106A at a bond distance of 1.933 and $1.229 \AA$ respectively and with $\operatorname{Arg} 322 \mathrm{~A}$ at bond distance 

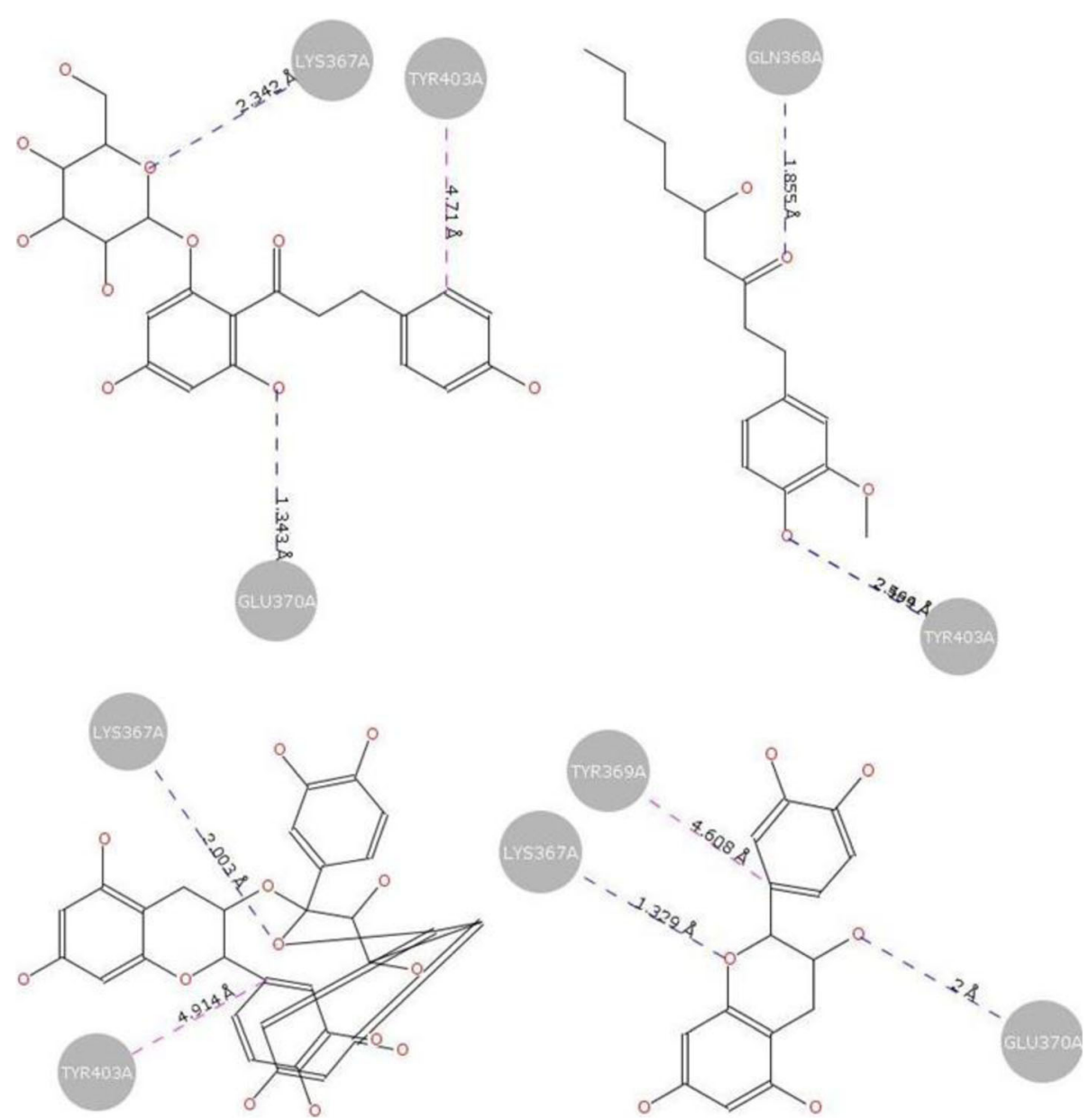

Fig. 7 Hydrogen bonding and aromatic interactions of phlorizin with cocrystallized ligand-tracked active site in 1N8S (top left). Hydrogen bonding of 6-gingerol with cocrystallized ligand-tracked active site in 1N8S (top right). Hydrogen bonding and aromatic interactions of procyanidin with cocrystallized ligand-tracked active site in 1N8S (bottom left). Hydrogen bonding and aromatic interactions of epicatechin with cocrystallized ligand-tracked active site in 1N8S

of 2.506, 2.499, and 2.494 $\AA$. 6-Gingerol has van der Waal's interactions with Ile85A, Pro93A, Arg96A, Tyr108A, Leu109A, Met226A, Ala227A, Val228A, Ser229A, His231A, His232A, Asp233A, Glu234A, and Arg322A while having hydrophobic interactions with Ile85A, Pro93A, Leu109A, Leu203A, Leu215A, Val228A, Ser229A, and His231A amino acid residues of active site of 4CXW. 6-Gingerol also has aromatic interactions with Tyr108A and His231A at a bond distance of 5.062 and $4.336 \AA$ respectively while having hydrogen bonding with Tyr106A, Glu234A, and Arg322A at a bond distance of $2.508,1.779$, and $2.573 \AA$ respectively. Procyanidin has van der Waal's interactions with Ile85A, Thr92A, Pro93A, Arg96A, Tyr108A, Leu109A, Ala227A, Val228A, Ser229A, Trp230A, His231A, His232A, Asp233A, Glu234A, and Arg322A while having hydrophobic interactions with Ile85A, Leu109A, Val228A, and His231A amino acid residues of active site of 4CXW. Procyanidin also has aromatic interactions with His231A at bond distance of 4.313 and $3.684 \AA$ while having hydrogen bonding with Ser229A, Trp230A, and Glu234A at a bond distance of 2.010, 2.245, and 1.519 respectively. Epicatechin has van der Waal's interactions with Thr92A, Pro93A, Val94A, Arg96A, Tyr108A, Leu109A, Ala227A, Val228A, Ser229A, His231A, His232A, Asp233A, and Glu234A while having hydrophobic interactions with Leu109A, Val228A, and His231A amino acid residues of active site of 4CXW. Along with that, epicatechin has aromatic interaction with His231A at a bond distance of $4.077 \AA$ while having hydrogen bonding with Arg96A and Glu234A at a bond distance of 2.587 and $2.477 \AA$ respectively. The reference ligand, (2E)-4-[N'-(4-benzyl-pyridine-3-carbonyl)-hydrazino]-4-oxo-but-2-enoic acid, has van der Waal's interactions with Arg96A, Tyr106A, Tyr108A, Leu109A, Leu203A, Asn205A, Val228A, His231A, His232A, Asp233A, Glu234A, Val244A, Tyr295A, His307A, Val309A, Arg316A, Ser318A, Thr320A, and Arg322A 
while having hydrophobic interactions with Tyr108A, Leu109A, Val228A, His231A, Asp233A, Glu234A, Val244A, Val309A, Ser318A, and Thr320A amino acid residues of active site of human alpha-ketoglutaratedependent dioxygenase FTO. This reference ligand also has charge interactions with Asp233A and Glu234A at a bond distance of 4.231 and $4.640 \AA$ respectively while having hydrogen bonding with His231A at bond distance of 1.923 and $2.195 \AA$ and with Glu234A at bond distance of 2.126 and $1.850 \AA$ and with His $307 \mathrm{~A}$, Arg316A, Ser318A, and Arg322A at a bond distance of 2.031, $2.259,2.028$, and $1.802 \AA$ respectively. Along with that

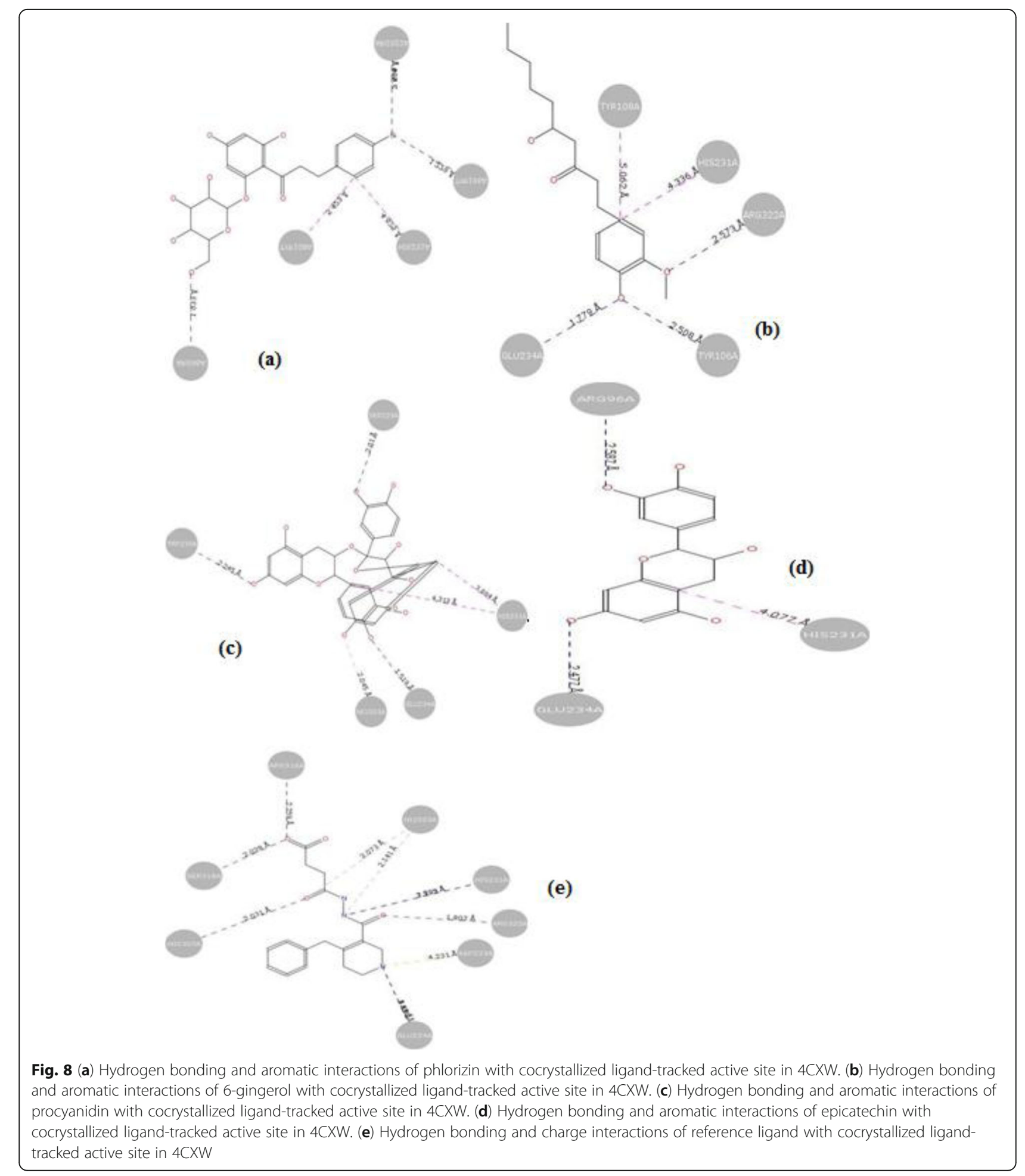


the reference ligand also has metal interactions with $\mathrm{Ni}^{2+}$ at a bond distance of 2.073 and $2.141 \AA$ (Fig. 8).

\section{Discussion}

Value added black tea or VABT prepared basing on the desirability function (Fig. 2) in the chemometrically optimized ratio of 420:50:54:26 showed the presence of several pharmacologically active molecules as per LC-MS results (Figs. 3, 4, and 5). Among the detected molecules include phlorizin, 6-gingerol, procyanidin, epicatechin (Figs. 3, 4, and 5) along with several other polyphenolics all lying in the combinatorial matrix of VABT. The pharmacologically active molecules detected in VABT showed significant potencies in inhibiting enzymes considered to have pathogenic effects in diabesity. In silico studies in revealing the versatile pharmacology of active molecules has also been carried out in corroborative research studies (Singla et al. 2016; Singla and Bhat, 2010a, b; Malleshappa and Harun 2011; Singla 2014; Igoli et al., 2014a, b, c, d; Igoli et al. 2014; Singla et al. 2012; Singla 2015; Singla et al. 2017; Sahu et al. 2017; De et al., 2017a, b; Srivastava et al. 2018; Srivastava et al. 2017). Phlorizin (phloretin-2'-O-glucoside) a dihydrochalcone is found to be a potent antioxidant, found to improve hyperglycemia by blocking renal glucose reabsorption and intestinal glucose absorption through inhibition of the sodium-glucose symporters, and also found to exert its protective effect against obesity (Cai et al. 2013; Shin et al. 2016). 6-Gingerol is an aromatic polyphenol and an important chemical constituent of ginger. This compound exhibited a multitude of antidiabetic and antiobesity effect by suppressing advanced glycation end products (AGEs) mediated rise in reactive oxygen species (ROS), significant insulinotropic activity mediated by glucagon like peptide 1 (GLP-1), and upregulating the critical components of GLP-1-mediated insulin secretion pathway viz. cyclic adenosine monophosphate (cAMP), protein kinase A (PKA), cAMP response elementbinding protein (CREB) (Son et al. 2015; Samad et al. 2017). Ginger supplementation in diet is found to suppress obesity (Ganapathy et al. 2014); ginger extracts were found to be ameliorative in obesity and inflammation by downregulating micro RNA-21/132 expression and activation of adenosine monophosphate activated protein kinase activity (AMPK) (Seunghae et al. 2018). Procyanidins are flavonoids with oligomeric structures known to alleviate oxidative stress and with insulinomimetic activity, also known to promote translocation of glucose transporter 4 (GLUT4), and activate AMPK signaling pathways (Pinent et al. 2004; Yamashita et al. 2016). Procyanidins can affect lipid synthesis, influence lipid degradation, adipose differentiation, and thus exert its protective role in obesity (Pinent et al. 2006). Dietary supplementation of epicatechin reduces adiposity and has effects on weight loss and weight maintenance (De et al. 2020; Hursel et al. 2009). In VABT, all potent compounds are present in the same matrix and is likely to exert a synergistic effect in alleviating diabesity as predicted by in silico studies and synergism supported by corroborative researches (De et al., 2017a, b).

\section{Conclusion}

In order to cope with the growing disease complexities, the medical fraternities have started promoting multitarget therapeutic entities. Dietary and lifestyle interventions have gained a strong focus as an adjuvant therapy. Phytonutraceuticals, food combinatorics, and herbosynthetic combinations are being increasingly applied as naturistic therapy to treat different chronic ailments, e.g., diabesity. The main purpose is to utilize their synergistic preventive and curative potentials so as to halt the disease at early stages and restrict further development of complications. Value addition in black tea is obvious to enhance its health potentials due to a multitude of pharmacologically active molecules present in its common matrix. In silico tools were found to be effective in predicting the activity of the compounds in VABT in combating diabesity; however further in vivo experimentations are warranted in this regard.

\section{Abbreviations \\ PSMs: Plant secondary metabolites; VABT: Value added black tea; RSM: Response surface methodology; ORAC: Oxygen radical absorbance capacity; ESI: Electrospray ionization; PDB: Protein Data Bank; MMFF: Merck Molecular Force Field; rms: Root mean square; ROS: Reactive oxygen species; AGEs: Advanced glycation end products; GLP-1: Glucagon-like peptide 1; CAMP: Cyclic adenosine monophosphate; PKA: Protein kinase A; CREB: CAMP response element-binding protein; AMPK: Adenosine monophosphate activated protein kinase activity; GLUT4: Glucose transporter 4; FTIR: Fourier transfer infrared spectroscopy; LC-MS: Liquid chromatography-mass spectrometry}

\section{Acknowledgements \\ The Sponsor National Tea Research Foundation (NTRF) is deeply acknowledged.}

\section{Author's contributions}

The central theme of the work was developed by B.De with K. Bhandari under the guidance of T.K. Goswami. Experimental works were done by B.De with K. Bhandari. In silico GRIP docking studies have been done by R. K Singla. G. Saha provided valuable inputs in developing the study protocol and data analysis. Manuscript was written by B.De, edited with K. Bhandari, and revised with T.K. Goswami. All authors read and approved the manuscript.

\section{Funding}

National Tea Research Foundation (NTRF), Tea Board of India.

Availability of data and materials

The data and materials during the study are available in the laboratory.

\section{Competing interest}

Authors declare that they have no competing interests.

Ethics approval and consent to participate

Not applicable. 


\section{Consent for publication}

Not applicable.

\section{Author details}

${ }^{1}$ Department of Agriculture and Food Engineering, Indian Institute of Technology, Kharagpur 721302, India. ${ }^{2}$ Institutes for Systems Genetics West China Hospital, Sichuan University, WuHou District, Chengdu, Sichuan 610041, China. ${ }^{3}$ National Tea Research Foundation, Tea Board of India, Kolkata, India.

\section{Received: 11 February 2020 Accepted: 17 April 2020} Published online: 01 May 2020

\section{References}

Belavic A (2015) Diabesity: Obesity as a risk for diabetes mellitus Type 2. 1-28.

Bhandari K, Singla RK, De B, Ghosh BC, Katakam P, Khushwaha DK, Gundamaraju R, Sen G, Saha G, Mitra A, Mitra A (2015) Chemometrics based extraction of polyphenolics from fresh tea leaves and processed tea showing in-silico docking and anti-oxidative theronostic dietary adjuvant in Alzheimer. Indo Glob J Pharm Sci 5(3):171-191

Cai Q, Li B, Yu F, Lu W, Zhang Z, Yin M, Gao H (2013) Investigation of the protective effects of phlorizin on diabetic cardiomyopathy in $\mathrm{db} / \mathrm{db}$ mice by quantitative proteomics. J Diabetes Res:1-9

De B, Bhandari K, Chakravorty N, Mukherjee R, Gundamaraju R, Singla RK, Katakam P, Adiki SK, Ghosh B, Mitra A (2015a) Computational pharmacokinetics and in vitro-in vivo correlation of anti-diabetic synergistic phyto-composite blend. World J Diabetes 6(11):1179-1185

De B, Bhandari K, Katakam P, Goswami KT (2019a) Development of a standardized combined plant extract containing nutraceutical formulation ameliorating metabolic syndrome components. SN Appl Sci 1:1484

De B, Bhandari K, Katakam P, Saha G, Goswami KT (2019b) Turmeric black tea as a multimodal theronostic dietary adjuvant aiding neuroprotection and ameliorating hypertension. MOJCRR 2(5):1-10

De B, Bhandari K, Singla RK, Katakam P, Samanta T, Kushwaha DK, Gundamaraju R, Mitra A (2015b) Chemometrics optimized extraction procedures, phytosynergistic blending and in vitro screening of natural enzyme inhibitors amongst leaves of Tulsi, Banyan and Jamun. Pharmacog Mag 11(44):522-532

De B, Singla RK, Bhandari K, Katakam P, Adiki SK, Mitra A (2017a) Study the enzyme inhibitory potentialities of a phytocomposite for type 2 diabetes by in silico GRIP docking. Int. J. Pharm. Integr. Life Sci 5:34-57

De B, Singla RK, Bhandari K, Katakam P, Adiki SK, Mitra A (2017b) GRIP docking studies to explore the enzyme inhibitory potentialities of a phytocomposite for type 2 diabetes. IJOPILS 5(3):34-57

De Los Santos S, Reyes-Castro LA, Coral-Vázquez RM, Méndez JP, Leal-García M, Zambrano E, Canto P (2020) (-)-Epicatechin reduces adiposity in male offspring of obese rats. J Dev Orig Health Dis. Feb 11(1):37-43.

Ganapathy S, Ponnusamy P, Machampalayam AD, Balasubramanian S (2014) Antiobesity action of gingerol: effect on lipid profile, insulin, leptin, amylase and lipase in male obese rats induced by a high-fat diet. J Sci Food Agric 94: 2972-2977

Ganguly S, Kumar T, Mantha S, Panda K (2016) Simultaneous determination of black tea derived catechins and theaflavins in tissues of tea consuming animals using ultra-performance liquid-chromatography tandem mass spectrometry. PLOS ONE 11(10):e0163498

Ghosh HN, Yang Won-Mo C (2015) Diversification of the tea products - global scenario. J Tea Sci Res 5(3):1-10

Hursel R, Viechtbauer W, Westerterp-Plantenga MS (2009) The effects of green tea on weight loss and weight maintenance: a meta-analysis. Int J Obes 33: 956-961

Igoli JO, Gray Al, Clements CJ, Kantheti P, Singla RK (2014a) Antitrypanosomal activity \& docking studies of isolated constituents from the lichen Cetraria islandica: possibly multifunctional scaffolds. Curr Top Med Chem 14:10141021

Igoli JO, Gray Al, Clements CJ, Kantheti P, Singla RK (2014b) Antitrypanosomal activity \& docking studies of isolated constituents from the lichen Cetraria islandica: possibly multifunctional scaffolds. Curr Top Med Chem 14:10141021

Igoli NP, Clements CJ, Singla RK, Igoli JO, Uche N, Gray Al (2014C) Antitrypanosomal activity \& docking studies of components of Crateva adansonii DC leaves: novel multifunctional scaffolds. Curr Top Med Chem 14: 981-990
Igoli NP, Clements CJ, Singla RK, Igoli JO, Uche N, Gray Al (2014d) Antitrypanosomal activity \& docking studies of components of Crateva adansonii DC leaves: novel multifunctional scaffolds. Curr Top Med Chem 14: 981-990

Jadhav K, Mukhopadhyay S, Ajmani S, Kakulte K, Kulkarni SA GRIP docking: a simple and fast docking method. VLife Sciences Technologies Pvt. Ltd.

Jawla S, Kumar Y (2013) Molecular docking interaction of Pinitol (ligand) with dipeptidyl peptidase 4 receptor (PDB 3C45). World Appl Sci J 24(12):1629_ 1634

Kaushik P, Khokra SL, Rana AC, Kaushik D (2014) Pharmacophore modeling and molecular docking studies on Pinus roxburghii as a target for diabetes mellitus. Adv Bioinformatics:1-8

Mahendran G, Manoj M, Murugesh E, Kumar RS, Shanmughavel P, Rajendra PK, Narmatha BV (2014) In vivo antidiabetic, antioxidant and molecular docking studies of 1,2, 8- tri hydroxyl-6-methoxy xanthone and 1,2 dihydroxy-6methoxyxanthone-8-O- $\beta$-D xylopyranosyl isolated from Swertia corymbosa. Phytomedicine 21:1237-1248

Malleshappa NN, Harun MP (2011) A comparative QSAR analysis and molecular docking studies of quinazoline derivatives as tyrosine kinase (EGFR) inhibitors: a rationale approach to anticancer drug design. J Saudi Chem Soc 17:361379

Malleshappa NN, Patel HM (2013) A comparative QSAR analysis and molecular docking studies of quinazoline derivatives as tyrosine kinase (EGFR) inhibitors: a rationale approach to anticancer drug design. J Saudi Chem. Soc 17(4): 361-379

McNaughton D (2013) 'Diabesity' down under: overweight and obesity as cultural signifiers for type 2 diabetes mellitus. Crit Public Health 23(3):274-288

Medagama AB (2015) The glycaemic outcomes of cinnamon, a review of the experimental evidence and clinical trials. Nutr J 14(108):1-25

Meruva AD, Subbaiah GV, Reddy KS (2014) Molecular docking studies of Xanthium indicum bioactive compounds with therapeutic targets of diabetes mellitus. Int J Pharm Sci Rev Res 29(1):236-240

Mitra A, Dewanjee D, Dey B (2012) Mechanistic studies of lifestyle interventions in type 2 diabetes. World J Diabetes 3(12):201-207

Mohamed GA, Ibrahim SRM, Elkhayat ES, Dine RSE (2014) Natural anti-obesity agents. Bull Fac Pharm Cairo Univ 52:269-284

Pinent M, Bladé C, Salvadó MJ, Blay M, Pujadas G, Fernández-Larrea J, Arola L, Ardévol A (2006) Procyanidin effects on adipocyte-related pathologies. Crit Rev Food Sci Nutr 46(7):543-550

Pinent M, Blay M, Bladé MC, Salvadó MJ, Arola L, Ardévol A (2004) Grape seedderived procyanidins have an antihyperglycemic effect in streptozotocininduced diabetic rats and insulinomimetic activity in insulin-sensitive cell lines. Endocrinology 145(11):4985-4990

Pokuri S, Singla RK, Bhat VG, Shenoy GG (2014) Insights on the antioxidant potential of 1,2,4-triazoles: synthesis, screening \& QSAR studies. Curr. Drug Metab 15:389-397

Sahu D, Sharma S, Singla RK, Panda AK (2017) Antioxidant activity and protective effect of suramin against oxidative stress in collagen induced arthritis. Eur J Pharm Sci 101:125-139

Samad MB, Mohsin Md NAB, Razu BA, Hossain MT, Mahzabeen S, Unnoor N, Muna IA, Akhter F, Kabir AU, Hannan JMA (2017) [6]-Gingerol, from Zingiber officinale, potentiates GLP-1 mediated glucose stimulated insulin secretion pathway in pancreatic $\beta$-cells and increases RAB8/ RAB10-regulated membrane presentation of GLUT4 transporters in skeletal muscle to improve hyperglycemia in Leprdb/db type 2 diabetic mice. BMC Complement Altern Med 17(395):1-13

Seunghae K, Lee MS, Jung S, Son HY, Park S, Kang B, Kim SY (2018) In-Hwan Kim, Chong-Tai Kim and Yangha Kim. Ginger extract ameliorates obesity and inflammation via regulating microRNA-21/132 expression and AMPK activation in white adipose tissue. Nutrients 10(1567):1-12

Shin S-K, Cho S-J, Un-Ju J, Ryu R, Choi MS (2016) Phlorizin supplementation attenuates obesity, inflammation, and hyperglycemia in diet-induced obese mice fed a high-fat diet. Nutrients 8(92):1-10

Simon MJ, Lagergren ES, Wathne LG (1999) Optimizing high-performance concrete mixtures using statistical response surface methods. In Proceedings of the 5th International Symposium on Utilization of High-Strength/HighPerformance Concrete. Norwegian Concrete Association, Oslo, Norway, June 1311-1321.

Singla RK (2014) Mechanistic evidence to support the anti-hepatitis B viral activity of multifunctional scaffold \& conformationally restricted magnolol. Natl. Acad. Sci. Lett 37:45-50 
Singla RK (2015) Homology modelling of MDR1 Gene MDR1_ENTHI of E. histolytica \& its molecular docking with anti-Entamoeba histolytica agents. Curr Top Med Chem 15:980-989

Singla RK, Bhat VG (2010a) QSAR model for predicting the fungicidal action of 1,2,4-triazole derivatives against Candida albicans. J Enzyme Inhib Med Chem 25(5):696-701

Singla RK, Bhat VG (2010b) QSAR model for predicting the fungicidal action of 1,2,4-triazole derivatives against Candida albicans. J Enzyme Inhib Med Chem 25:696-701

Singla RK, Bhat VG, Kumar TNVG (2013) 3D-quantitative structure activity relationship: a strategic approach for in silico prediction of anti-candididal action of 1, 2, 4-triazole derivatives. IGJPS 3(1):52-57

Singla RK, Paul P, Nayak PG, Bhat VG (2012) Investigation of anthramycin analogs induced cell death in MCF-7 breast cancer cells. Indo Global J Pharm Sci 2: 383-389

Singla RK, Scotti L, Dubey AK (2017) In silico studies revealed multiple neurological targets for the antidepressant molecule ursolic acid. Curr. Neuropharmacol 15:1100-1106

Singla RK, Singh R, Dubey AK (2016) Important aspects of post-prandial antidiabetic drug. Acarbose Curr Top Med Chem 16:2625-2633

Son MJ, Miura Y, Yagasaki K (2015) Mechanisms for antidiabetic effect of gingerol in cultured cells and obese diabetic model mice. Cytotechnology 67:641-652

Srivastava V, Singla R, Dubey A (2017) Inhibition of secretory aspartyl protease of Candida albicans by metabolites of Streptomyces chrestomyceticus strain ADP4. In Proceedings of the MOL2NET, International Conference on Multidisciplinary Sciences, 15 January-15 December; Sciforum Electronic Conference Series, 3, doi:https:/doi.org/10.3390/mol2net-03-04969.

Srivastava V, Singla RK, Dubey AK (2018) Inhibition of biofilm and virulence factors of Candida albicans by partially purified secondary metabolites of Streptomyces chrestomyceticus strain ADP4. Curr Top Med Chem 18(11): 925-945

V Life MDS: Molecular Design Suite, V Life sciences technologies Pvt. Ltd., Pune, India, 2013 (www.vlifesciences.com)

Yamashita Y, Wang L, Nanba F, Ito C, Toda T, Ashida H (2016) Procyanidin promotes translocation of glucose transporter 4 in muscle of mice through activation of insulin and AMPK signaling pathways. PLoS ONE 11(9):e0161704

\section{Publisher's Note}

Springer Nature remains neutral with regard to jurisdictional claims in published maps and institutional affiliations.

\section{Submit your manuscript to a SpringerOpen ${ }^{\circ}$ journal and benefit from:}

- Convenient online submission

- Rigorous peer review

- Open access: articles freely available online

High visibility within the field

- Retaining the copyright to your article

Submit your next manuscript at $\boldsymbol{\nabla}$ springeropen.com 